\title{
JARDINES INFANTILES COMUNITARIOS EN CHILE: UNA HISTORIA DE RESISTENCIA
}

\author{
Graciela Muñoz Zamora ${ }^{1}$
}

\begin{abstract}
RESUMEN: Los avances de la educación de la primera infancia han sido relevantes en América Latina, producto de los consensos de los gobiernos por garantizar la atención y una educación de calidad para los niños y niñas. Chile se ha destacado por la construcción de una institucionalidad y por el alto nivel de cobertura. No obstante, existen experiencias comunitarias de atención y educación a la primera infancia que han sido invisibilizadas y que se vinculan al movimiento de pobladoras de la década de los ochenta. Esta investigación se desarrolló durante dos años y buscó conocer la historia y procesos que han desarrollado cinco Jardines Comunitarios. Su historia se vincula a una resistencia en el contexto neoliberal de la educación chilena.
\end{abstract}

Palabras-clave: Primera infancia. Jardín infantil. Comunidad. Identidad. Resistencia.

\section{COMMUNITY KINDERGARTENS IN CHILE: A HISTORY OF RESISTANCE}

\begin{abstract}
The development of early childhood education has been relevant in Latin America, as a result of a consensus by the governments in order to guarantee service and quality education for boys and girls. Chile has been important in the region, due to its development in childhood education, driven by a growing institutionalization and an expansion in coverage. However, some experiences in early childhood education, connected to the slum community women in the 80 's, have been made invisible. Our research was conducted for two years, aiming at gaining knowledge about the history and the processes developed by five Community Kindergartens. Their history appears to be connected to a sense of resistance within the neoliberal context of the Chilean education.
\end{abstract}

Keywords: Early childhood. Kindergartens. Community. Identity. Resistance.

*Este artículo de investigación forma parte de un estudio más amplio financiando por la Dirección de Investigación de la Universidad Metropolitana de Ciencias de la Educación: FGI 1016, "Imaginarios de la Infancia: Una Mirada desde la Formación Docente”.

1.Universidad Metropolitana de Ciencias de la Educación - Facultad de Filosofía y Educación - Departamento de Educación Parvularia - Santiago, Chile. E-mail: graciela.munoz@umce.cl 


\title{
JARDINS DE INFÂNCIA COMUNITÁRIOS NO CHILE: UMA HISTÓRIA DE RESISTÊNCIA
}

\begin{abstract}
RESUMO: Os avanços da educação na primeira infancia foram relevantes na América Latina, resultado do consenso dos governos para a garantia da atenção e de uma educação de qualidade para os meninos e meninas. O Chile tem se destacado pela construção de uma institucionalidade e pelo alto nível de cobertura. Contudo, existem experiências comunitárias de atenção e educação à primeira infância invisibilizadas e que se vinculam com o movimento das moradoras da década de 1980. Este estudo se desenvolveu durante dois anos e buscou conhecer a história e os processos de desenvolvimento de cinco jardins comunitários. Essa história se vincula a uma resistência no contexto neoliberal da educação chilena.
\end{abstract}

Palavras-chave: Primera infância. Jardim de infância. Comunidade. Identidade. Resistência.

\section{La Convivencia de Experiencias Comunitarias e Institucionalizadas en América Latina}

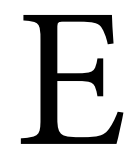

n general, existe consenso en América Latina sobre que la educación de la primera infancia es un derecho humano universal y que los gobiernos deben generar políticas para garantizarlo. Sin embargo, el problema en la región es principalmente la cobertura y los recursos que se destinan a su desarrollo, pues estos últimos son insuficientes para cubrir las necesidades de una educación de calidad para la primera infancia. Los gobiernos, para financiar estos programas, deben recurrir a organismos internacionales, como el Banco Mundial y el Banco Interamericano de Desarrollo. Según Berlinski y Schady (2015), la política de estos organismos internacionales se ha orientado al aumento de la cobertura, "mediante provisión pública, o indirecta, financiando y regulando a los proveedores privados" $(2015$, p. 186). En el caso de los proveedores privados, se realiza a través del financiamiento a través de un sistema de voucher o subvención, que pone en marcha una privatización encubierta de la educación (BALL; YOUDELL, 2008). Esto tensiona la educación pública en cada país.

En la región, históricamente se han desarrollado experiencias comunitarias de atención y educación a la primera infancia, las que datan desde 1965, en Puno, Perú, y en Argentina, en el año 1930. En general, han sido experiencias rurales, muchas de las cuales han surgido desde la organización y la movilización de la comunidad, atendidas por madres y jóvenes. Peralta (2018) menciona que después se integraron ONG y fundaciones benéficas y organismos internacionales ${ }^{1}$ para apoyar el desarrollo de esas experiencias o instalar programas nuevos. Estos programas han evidenciado la necesidad de incorporar una política sistemática para el desarrollo de la atención y educación infantil en la región.

En la actualidad, en un estudio realizado por el BID, se señala que en América Latina hay una heterogeneidad de programas y servicios que atienden a la primera infancia que pueden ser institucionalizados o comunitarios. En sectores rurales, sin embargo, predominan programas comunitarios de apoyo parental, los que entregan servicios de corte nutricional y pedagógico (ARAUJO et al., 2013). También existen experiencias que tienen un componente comunitario y que lograron institucionalizarse, como es el caso de Colombia, con los Hogares Comunitarios de Bienestar (HCB, de 1974), que surgieron para responder a la situación de violencia que sufrían los niños en los sectores urbanos. En Costa Rica, desde 1949, están los Centros de 
Educación y Nutrición (CEN) y Centros Infantiles de Nutrición y Atención Integral (CINAI), programas de apoyo parental que funcionan desde los centros de salud. Finalmente, Uruguay tiene el Plan Centros de Atención a la Infancia y Familia (CAIF, de 1987), un programa piloto experimental de Unicef que entrega servicios de nutrición y atención integral. La institucionalización de estos programas, con un enfoque asistencialista y de estimulación temprana, cambia para asumir un enfoque educativo. Todo lo anterior favoreció la ampliación de cobertura en cada uno de los países.

Sin embargo, en algunos países fueron los programas no formales o comunitarios los que tuvieron una mayor aceptación y se expandieron con mayor rapidez que los programas formales o institucionalizados (KAMERMAN, 2006). Algunas de esas experiencias, como en el caso argentino, vienen desde el año 1930, con programas de guardería y atención preescolar comunitaria. En la misma línea se reconocen los Hogares Comunitarios en Colombia. En la década de 1990, Guatemala y Perú promovieron los programas comunitarios; Honduras implementó un programa nacional (Programa Atención Integral a la Niñez-AIEPI-AIN-C); Nicaragua creó un Programa de Atención Integral a la Niñez (PAININ) a nivel nacional y en Bolivia se inició el Programa de Apoyo Parental mediante encuentros semanales para niños indígenas (ARAUJO et al., 2013). Muchos de estos programas surgieron desde la gestión de las comunidades y luego fueron administrados por la institucionalidad central o municipal para lograr una mayor cobertura, siempre manteniendo el vínculo con la comunidad.

Las experiencias comunitarias desarrolladas en América Latina buscan "ampliar la atención a la niñez con un menor costo que la vía institucional, alcanzando grados de incidencia creciente y resultando una deseable respuesta educativa para sectores sociales que se encuentran en una situación desfavorecida" (ANCHETA; LÁZARO, 2012, p. 116). Sin embargo, estas propuestas han sido cuestionadas en su efectividad según criterios de calidad esgrimidos por la institucionalidad, como la dotación y la calificación del personal, condiciones de infraestructura y recursos, materiales didácticos, seguridad y presencia de entidades fiscalizadoras, entre otros. Con todo, estas iniciativas develan el papel limitado del Estado en su capacidad de garantizar el derecho y la atención a la primera infancia y "cobran protagonismo de entidades no gubernamentales de la sociedad civil” (RAMÍREZ OTÁLVARO, 2015, p. 34).

Estos programas tienen una base teórica crítica, muy permeada por el pensamiento de Paulo Freire, ya que surgen de contextos comunitarios y territoriales concretos centrados en las necesidades de los niños y niñas y con participación parental (FUJIMOTO-GÓMEZ, 2000). Algunas de estas experiencias han sido valoradas positivamente por el Estado y otras, por el gobierno local, debido principalmente a la pertinencia cultural y participación de las familias y comunidad, lo que ha favorecido la inclusión de los niños y niñas más pobres de la sociedad, extendiendo su acción a las familias que han entendido la importancia del cuidado y la educación. También se reconoce que estas propuestas han contribuido al desarrollo local de las comunidades y al mejoramiento de las condiciones de vida. ALBORNOZ (2012) menciona que estas experiencias han logrado instalar un proyecto pedagógico que se encuentra al servicio de un proyecto político para el desarrollo de las comunidades, y genera un proceso de restitución de derechos en la comunidad. Estas experiencias, en opinión de Young y Fujimoto-Gómez (2004), responden a concretar una política de igualdad de oportunidades y equidad en lo local, en que el componente participativo es clave; sin embargo, es un aspecto ausente en algunas políticas educativas en la región.

\section{La Educación de la Primera Infancia en Chile}

En Chile, la educación infantil ha tenido prioridad en la política pública, especialmente con el reconocimiento del nivel en la Ley General de Educación (Ley n. 20.370) y su integración al Sistema de 
Aseguramiento de la Calidad Educativa (Ley n. 20.259). Esto se ha reflejado en medidas que tienden a institucionalizar la educación parvularia y a instalar procesos de calidad educativa para todas las instituciones que atienden a la primera infancia. Actualmente, por una parte, se creó la Subsecretaria de Educación, encargada de establecer políticas para la educación del nivel, y, por otra, la Intendencia de Educación Parvularia, cuyo objetivo es proponer normas de fiscalización para avanzar en la calidad educativa. ${ }^{2}$ Junto con esto, se busca profesionalizar la labor de los educadores infantiles y mejorar la calidad del nivel educativo a través de los Estándares para la formación de educadoras de párvulos, Marco de Buena Enseñanza, entre otros dispositivos para ese objetivo (MINEDUC, 2016).

En Chile, la Junta Nacional de Jardines Infantiles (JUNJI), creada en 1970, es reconocida pionera en América Latina. Su desarrollo ha estado marcado por políticas centralistas; no obstante, ha dado un fuerte impulso al desarrollo de la educación infantil en la región. Otra de las instituciones relevantes es la Fundación Integra, de origen asistencialista, instalada por la dictadura militar, a través del Programa de Centros Abiertos y administrados por la Fundación Nacional de Ayuda a la Comunidad (FUNACO), creada en 1975. Su acción se focalizaba en entregar alimentación y cuidado. ${ }^{3}$ En los años 1990, en democracia, como parte de la política de Igualdad de Oportunidades, cambia su nombre por Fundación Integra, iniciando un proceso de profesionalización y contando con una red importante de Salas Cunas y Jardines Infantiles y modalidades no convencionales en todo el país.

En la actualidad, bajo la Ley General de Educación y el Sistema de Aseguramiento de Calidad, los Jardines Infantiles deben cumplir con las mismas exigencias que el resto de los niveles educativos, lo que se traduce en requisitos legales y pedagógicos para obtener el reconocimiento oficial y funcionar como tales. Entre ellos, deben contar con el Proyecto Educativo Institucional, reglamentos para el funcionamiento, personal idóneo, acreditar el local y el capital, entre otros. Esto, sin duda, tensiona algunas instituciones que atienden a la primera infancia. ${ }^{4}$

Por otra parte, no se puede desconocer el impulso en cuanto a cobertura dado desde el primer gobierno de Michelle Bachelet. Según cifras de la Subsecretaria de Educación Parvularia (2017), en el primer y segundo nivel transición (5 a 6 años), existe casi en una cobertura universal con un 90, 77 y 98,26\% respectivamente, atendido por establecimientos educacionales de distintas dependencias (municipales, particulares subvencionados y particulares pagados). En cambio, en el nivel medio ( 3 a 4 años), la cobertura alcanza un $48,84 \%$ y en sala cuna, $18,73 \%$, que comprende jardines infantiles pertenecientes a instituciones públicas y privadas.

El aumento de la cobertura en Chile se ha realizado a partir del subsidio a la demanda. ${ }^{5}$ Si bien esta política neoliberal ${ }^{6}$ se instaló en el gobierno militar, ha tenido una continuidad con los gobiernos en democracia. Este subsidio opera a través de convenios: en el caso de Integra, a través de Convenios de Administración Delegada y, en el caso de JUNJI, a través de Vías de Transferencia de fondo (VTF). Ambos entregan financiamiento a organizaciones públicas, como las corporaciones municipales, que administran la educación o Fundaciones y Corporaciones privadas sin fines de lucro, como el modo elegido para lograr los aumentos de la cobertura (TOKMAN, 2010). Esto dificulta el rol del Estado en garantizar el derecho a la educación de la primera infancia, ya que no asegura un servicio en igualdad de condiciones, puesto que los recursos se reciben por la asistencia de los niños y niñas a los centros educativos, y no garantizan un aporte basal para el funcionamiento de los centros, a diferencia de los jardines infantiles y salas cunas, administradas directamente por JUNJI y Fundación Integra.

\section{Programas no Convencionales en Chile}

En Chile, el trabajo comunitario con la primera infancia se desarrolló principalmente en la dictadura militar, a través del movimiento de pobladores e iniciativas desarrolladas por Fundaciones como MISSIO 
y ONG como PIIE, CIDE y CEANIM, las que apoyaron iniciativas surgidas desde la propia comunidad y otras creadas por ellas. Estas experiencias son conocidas como programas no formales (PERALTA, 2018).

Desde 1990, en JUNJI, existe el Programa Educativo Alternativo de Atención al Párvulo (PA).7 Su objetivo es acercar una oferta educativa en zonas de dispersión geográfica, entregando una oferta alternativa y flexible para responder a las características de las comunidades y territorios rurales y urbanos de alta vulnerabilidad social, promoviendo el bienestar integral en niños y niñas de 2 a 5 años 11 meses de edad. Estos programas aplican planificación curricular para apoyar la labor que realiza el personal y favorecen una relación directa con las familias y la comunidad. En una evaluación realizada por la DIPRES (2017), se menciona que ha ido aumentando en cobertura gracias a la difusión de la importancia del nivel; sin embargo, debe "definir y operacionalizar el concepto de pertinencia y alternativo para que esto no signifique entregar educación parvularia semejante a la convencional, pero con menores estándares de calidad” (2017, p. 77). Esta frase da cuenta de la tensión existente entre "calidad" y "pertinencia cultural", en que el primer concepto tiene un enfoque tecnocrático sin dialogar con estas modalidades; en cambio, el segundo implica desarrollar criterios distintos para evaluar estos programas. No se puede desconocer que los aportes de estas iniciativas han sido significativos para la educación de los niños y niñas en sectores urbanos pobres y en sectores rurales, ya que su trabajo se vincula al rescate del patrimonio y la identidad y favorece la participación de organizaciones y agentes comunitarios.

Algunos de los Jardines Infantiles Comunitarios surgieron en el gobierno de Salvador Allende y en la dictadura militar. Sin embargo, el sistema educativo no los reconoce bajo esta denominación. ${ }^{8}$ La historia que han construido los Jardines Infantiles Comunitarios permite cuestionar el peso de la institucionalización presente en la educación infantil en Chile, su homogenización de criterios y los programas aplicados en los centros educativos. Por ello se requiere visibilizar una historia construida al margen y vinculada a la historia social del país. Estos centros actualmente son parte de la política de cobertura y han debido establecer convenios con JUNJI e Integra para contar con financiamiento; se les conoce como jardines de administración delegada (Integra) o Vías de Transferencia de Fondo (VTF) para JUNJI, lo que los convierte en jardines convencionales, pero sin duda su experiencia se debe visibilizar desde el aporte que han realizado con sus comunidades y dentro de ellas.

\section{Metodología}

La investigación fue desarrollada en un período de dos años, concretándose en cinco jardines comunitarios de la Región Metropolitana. El objetivo de la investigación era conocer su historia y su proyección futura.

Se realizó a través del método de estudio de casos (STAKE, 1998). Se aplicaron entrevistas en profundidad a distintos actores. Los sujetos entrevistados fueron 10 educadoras de párvulos con más 10 años de ejercicio en el centro (SujEd); 10 agentes comunitarios (SujP) y cinco directivos de la Corporación (SujD). Todos los participantes firmaron el consentimiento ético.

Al ser un estudio cualitativo, la voz de los sujetos es relevante para resguardar el sentido de la historia en la cual ellos han sido los protagonistas. Las categorías surgieron de dos etapas de análisis:

1. Intra-caso: permitió reconstruir la historia de cada uno de los centros que participaron del estudio y que fue validada por los entrevistados.

2. Análisis inter-caso: permitió determinar las categorías más representativas que dieran cuenta del proceso de los jardines infantiles que participaron en el estudio.

A continuación, se detallan las principales categorías. 


\section{Jardines Comunitarios Vinculados al Movimiento de las Pobladoras}

La mayoría de estos centros educativos surgieron entre los años 1970 y 1980, durante la dictadura militar. Su nacimiento se vincula con la pobreza y la crisis económica producidas por la instalación de políticas de corte neoliberal (SALAZAR, 2013; GARCÉS, 2003). Surgieron de la organización de los pobladores y, en algunos casos, desde las tomas de terrenos:

Se decide que dentro del espacio de la toma de terreno se iba a dejar un espacio para hacer un lugar de atención y de protección a los niños, porque la mujer organizada que participaba en las organizaciones sociales necesitaba un espacio donde los niños pudieran estar protegidos (SujEd1).

La situación económica era precaria y de pobreza; por tanto, desde la organización de las pobladoras, se fueron dando respuesta estas necesidades: "Había mucha pobreza; también las ollas comunes fueron un espacio que también vino a solucionar la pobreza y el hambre, que había en esos tiempos" (SujD3).

En estos espacios, el rol protagónico lo tienen las mujeres, quienes aportaron su trabajo para levantar espacios modestos donde atender a los niños y niñas: “[...] primero instalan unas mediaguas que eran espacios donde las mismas mujeres se turnaban y cuidaban a los niños" (SujD1). La principal motivación fue colaborar con la mantención de sus familias. En este tiempo surgieron las "ollas comunes" y el "comprando juntos", iniciativas todas gestadas por las mujeres pobladoras.

"Las mujeres sobre todo necesitaban poder salir a buscar el alimento y apoyar en el fondo a sus parejas o sus maridos, para poder mantener a sus familias, y generalmente las familias bastante numerosas [...] había harto niño solo" (SujEd8). También estaba presente la aspiración a que las mujeres se educaran junto con hijos: "[...] la idea de este proyecto era que la madre se educara, o el niño se educara junto a su madre; también era por sacar a la mujer de la casa” (SujD1). El papel de las mujeres en la dictadura fue protagónico en el desarrollo de los Jardines Infantiles Comunitarios, por los "problemas privados que se transformaron en problemas sociales" (VALDÉS; WEINSTEIN, 1989, p. 5). La organización les permitió movilizar y dinamizar a la comunidad, apoyadas desde la iglesia y las ONG, frente a la ausencia del Estado e impactadas por las consecuencias del modelo económico impuesto.

Lo anterior también se notó en la división sexual del trabajo y el imaginario social sobre el "cuidado" como responsabilidad femenina y, desplegándose en el contexto comunitario, específicamente en lo que respecta al cuidado infantil. Sin embargo, desde las teorías subalternas (FRASER, 1997), se puede reconocer que estas mujeres se fueron configurando como un actor social, específicamente en la visión política del trabajo con la infancia y el territorio, constituyéndose dentro del espacio público.

\section{Los Derechos de los Niños y Niñas son Derechos Humanos: Compromiso Vinculado a su Historia}

La acción de estos Jardines Comunitarios estuvo comprometida con el movimiento de resistencia a la dictadura en Chile y con la defensa de los Derechos Humanos del sector. Ellos fueron testigos de las jornadas de protesta nacional y se vinculan con la recuperación de la democracia como prioridad de acción política.

De esta manera, la integración de los Derechos Humanos al proyecto, cuyas prácticas educativas priorizan la afectividad y el vínculo, es un motor importante de la acción pedagógica, vinculando a las familias 
y la comunidad "[...] para nosotros lo más importante es servir con amor y darle ese amor a los niños que a veces no tienen afuera, porque tienen diferentes situaciones en sus hogares” (SujEd4).

En la sociedad chilena, los niños y niñas son invisibilizados, lo que profundiza su pobreza y marginalidad. Sin embargo, los educadores logran darles protagonismo contextualizando el trabajo de aula. De esta manera, cuestionan la relación de poder entre niños y adultos y sus propios imaginarios respecto de la infancia. Se puede afirmar que en su práctica los Jardines Comunitarios han comprendido que:

La Convención de los derechos niños y niñas ha abierto la posibilidad de reconocer -sin recortes- la dignidad de cada ser humano, de cada niño, niña; de respetar su identidad; de asumir que cada criatura es un ser público, es decir es de interés superior de la humanidad el que está en juego cada niño, niña. Pero además el enfoque de derechos ha desnaturalizado todo intento de justificar la sumisión, el trato discriminatorio, la exclusión de cualquier niño, niña, la impunidad frente a la violación de su dignidad, etc. (CUSSIÁNOVICH; FIGUEROA, 2009, p. 87).

Desde esta perspectiva, su acción no se limita solo al aula, sino que también a promover "comunidades protectoras de los derechos de la niñez", apoyando una cultura que valoriza a la infancia y la integra a la vida comunitaria, a través de la realización de caminatas por la población y de la participación en los aniversarios de la misma, lo que entrega una perspectiva política y ética respecto a la niñez, en que la prioridad de las acciones educativas y comunitarias están asentadas en el bienestar y desarrollo integral de los niños y niñas (REIS MONTEIRO, 2008).

\section{Proyecto Pedagógico Situado en su Realidad}

El surgimiento de los Jardines Comunitarios se vincula a una acción política y social del movimiento de pobladores, cuyo denominador común es la organización, la solidaridad y la identidad social: "Fuimos conociéndonos todos aquí, la toma fue en noviembre-diciembre del 72; nadie se conocía acá, estuvimos viviendo en carpas. Yo llegué en enero del año 73 [...]" (SujP1). Los cinco centros educativos han surgido junto con la población y han sido testigos de las transformaciones de su comunidad.

Esta acción política para generar un espacio para los niños y niñas se fue apropiando de la historia de cada uno de los sectores e integrando sus propios valores a la construcción de proyectos pedagógicos-políticos para la niñez y su comunidad, asentada en la historia local. De este modo, la historia local es referida en los procesos educativos de niños y niñas, lo que genera identidad. Así, algunas de las actividades fundamentales de los Centros están relacionadas con las celebraciones de aniversario de la población, articulando acciones con las organizaciones sociales y favoreciendo la participación de monitores comunitarios en el aula.

Una de las características importantes es el recate de la historia, el no olvidar un poco la misión para lo cual nació nuestro Jardín Infantil, que no solo es un jardín infantil, sino que tratamos de resolverle, resolver también en conjunto con la comunidad los problemas que hay respecto a infancia $[\ldots]$ un proyecto que está ligado a la comunidad (SujEd7).

Los centros son reconocidos y valorados por la comunidad: "El jardín es de nosotros, es patrimonio propio y respetado por los pobladores" (SujEd5). Los cinco jardines han construido una historia en conjunto con la comunidad, realizando acciones que van más allá de la acción educativa. En el presente, se encuentran 
abiertos a la comunidad y propician actividades con mujeres y con jóvenes; de esta manera, su presencia en la vida comunitaria se legitima.

No obstante, el modelo neoliberal fue impactando en las relaciones en los sectores populares, provocando la pérdida del tejido social y su capital comunitario y la pérdida de valores como la solidaridad y la identidad social, instalándose la apatía, el individualismo, la pérdida de las organizaciones y el surgimiento de problemas sociales (SOLIMANI, 2012; MUÑOZ, 1999). Además, el narcotráfico, la droga y delincuencia impactan en el aula porque es la realidad que vive la infancia en esos territorios, en los que hay que activar protocolos y dispositivos de seguridad para los niños y niñas "[...] les conversamos a los niños de que hay que tirarse al suelo cuando hay balacera [...]” (SujEd5).

El consumismo y el individualismo que se vive en los territorios han provocado una desesperanza, como lo expresan algunos de los pobladores más antiguos, ya que no cuentan con referentes o líderes en la comunidad que puedan mostrar valores y formas de vida distintos. Incluso, se menciona el vacío político local: "Los antiguos no han cambiado mucho, pero como la nueva generación que es diferente [...] estas generaciones no tiene lideres; antes teníamos ejemplos, ahora a quién” (SujP4). A pesar de esta situación, los jardines comunitarios no han renunciado a ocupar el espacio público con los niños y niñas y a través de las distintas actividades que realizan han aportado a recuperar el tejido social. Esto les permite ser valorizados por la comunidad, como se menciona:

[...] el jardín yo creo que es una fortaleza, es una historia. Cuando yo digo jardín infantil en tal población, donde sea, puede haber un jardín infantil, pero es diferente cuando tú hablas en la población del Jardín; no, el jardín es de nosotros, es algo patrimonio propio y respetado por los pobladores; aquí lo que ha pasado es que se les ha enseñado un poco a apropiarse del lugar (SujP8).

La praxis social y pedagógica de los Jardines Comunitarios les ha permitido legitimarse en sus comunidades pues han identificado problemáticas y necesidades de los territorios marginados y han desarrollado un proyecto situado y contextualizado. En palabras de Cabaluz (2016), estas experiencias han erigido "una pedagogía territorializada", reconocida por los propios pobladores como parte del patrimonio vivo de la comunidad, que valora el saber fuera de sus muros (CULLEN, 2008).

Un referente importante en la historia de estos jardines infantiles es la educación popular, que ha generado procesos de transformación social. Su acción se constituye en una opción ético-política que sus educadores y pobladores han logrado fundar a partir de la generación de prácticas sociales que promueven la solidaridad, la responsabilidad y la indignación frente a las injusticias; todo ello ha conformado la historia de los sectores populares (CABALUZ, 2016; FREIRE, 1993). Desde este reconocimiento de la dignidad de los sujetos, frente a las condiciones de exclusión, los jardines comunitarios han logrado generar espacios de esperanza en sus territorios (CULLEN, 2004; PINTO 2007).

\section{El Aprendizaje para Llegar a un Enfoque Educativo}

En sus inicios, los centros tenían un enfoque asistencial: el objetivo era cubrir las necesidades de alimentación y cuidado de los niños y niñas, mientras sus madres trabajaban. Sin embargo, han transitado hacia el desarrollo de una perspectiva de educación integral que les permite construir un currículo situado.

Los centros estudiados vivieron un proceso común: fueron sistematizando sus prácticas pedagógicas y generaron procesos de reflexión materializándolos en proyectos educativos innovadores en contextos de 
pobreza. Las experiencias educativas que desarrollan buscan ampliar el mundo cultural de niños y niñas, generando oportunidades para las familias y comunidad. Es por ello que el perfil del personal debe ser coherente con los objetivos: "Las educadoras tienen que tener una capacidad humana para estar aquí" (SujEd3), reconociendo que deben tener una opción clara para trabajar en esos contextos. Sus proyectos educativos centrados en el paradigma del buen vivir desarrollan metodologías que colocan en el centro a los niños y niñas, integrando elementos de la metodología Montessori, Pickler, biodanza, educación ambiental, educación popular, entre otros. Muchas de estas experiencias innovadoras suelen desplegarse en contextos socioeconómicos distintos en nuestro país. En sus aprendizajes, los jardines comunitarios han logrado ir mirando el contexto socio cultural, la memoria social y la pedagogía infantil, desarrollando una experiencia que les ha permitido sostener un discurso educativo propio (REDONDO, 2012).

Los agentes y monitores comunitarios se han integrado en la labor educativa a través de la realización de talleres, pero también se reconoce la labor de educadores naturales, muchas de ellas madres, que lograron estudiar y volvieron a trabajar al jardín comunitario, construyendo así una visión compartida. (FULLAN; HARGREAVES, 2000)

\section{El Tira y Afloja de la Institucionalización}

Con la entrada a la democracia, Chile dejó de ser un país prioritario para la cooperación internacional, aunque las necesidades seguían estando presentes en las poblaciones. También aparecen presiones legales que llevaron a muchas organizaciones a cumplir con la Ley de Asociatividad y Participación Ciudadana, constituyéndose en Corporaciones para acceder a los fondos públicos (GRUNNINGER, 2003). Lo anterior se transforma en una estrategia de sobrevivencia.

Este aspecto también impactó a los jardines comunitarios, que se transformaron en corporaciones o fundaciones para acceder a recursos proporcionados por el Estado. Esto les permitió beneficiarse de la ampliación de la política de cobertura, a través de las transferencias de fondos realizados por JUNJI y Fundación Integra. Estas instituciones los subvencionan como prestadores de servicios por la asistencia de los niños y niñas. Lo perverso del sistema es que la asistencia disminuye notoriamente en los meses de invierno debido a las enfermedades respiratorias. Los directores de los jardines infantiles comunitarios afirman que "administran la pobreza", ya que muchas veces no cuentan con recursos para pagar los sueldos al personal. La desigualdad y discriminación se manifiesta frente a los jardines infantiles de dependencia JUNJI e Integra que cuentan con un financiamiento basal. Uno de los actores menciona: "Somos los parientes pobres de la educación parvularia” (SujD3). Esta política de corte neoliberal consolida un financiamiento para pobres que aumenta las brechas económicas con los jardines infantiles JUNJI y de Integra, lo que se refleja claramente en la frase "administrar la pobreza".

Otro aspecto que han debido sortear con las financiadoras son los requerimientos técnicos y pedagógicos que les solicitan, contradictorios con su proyecto educativo como, por ejemplo, las normativas y la burocracia de procesos cotidianos, como los permisos para realizar paseos o visitas a la comunidad, o las restricciones para solicitar colaboración a las familias, entre otras. En opinión de las educadoras, la relación con la institucionalidad es vivida como una falta de empatía, ya que son criterios que no responden a su realidad y que homogenizan los procesos educativos. A pesar de esto, algunos han logrado resistir e incidir en la relación con los supervisores para dialogar y generar consensos y así lograr cambios en las miradas y acciones que recomiendan instalar. Lo anterior anticipa futuras 
tensiones con la aplicación del Sistema de Aseguramiento de la Calidad Educativa, ya que deberán asumir una serie de requisitos para cumplir con los criterios de calidad.

Toda esta gama de presiones y el requerimiento de institucionalizarse impiden el vínculo con la comunidad y tienden, entonces, a encerrarse, considerando el "afuera" como peligroso.

\section{Consideraciones Finales}

La historia y trayectoria de los cinco Jardines Infantiles comunitarios iluminan una lectura actual del contexto neoliberal de la educación en Chile que tensiona la educación de la primera infancia.

En la historia de estos Jardines Infantiles se aprecia un proceso de respuesta a las necesidades de los niños y niñas a través de la acción organizada de las mujeres pobladoras para construir proyectos educativos autónomos, contextualizados en lo local y que propician un compromiso con los derechos de los niños y niñas y sus comunidades. En este sentido, los educadores han realizado una lectura de su realidad para construir un proyecto de transformación política y educativa. Desde esta perspectiva, los jardines comunitarios han sabido dialogar con su realidad, recuperando las voces y los saberes de las distintas personas de la comunidad, y son clave para potenciar un protagonismo de los sujetos y promover la participación social (MEJÍA, 2013), lo que se traduce en su Proyecto Educativo. Sin embargo, las políticas educativas neoliberales y los criterios de calidad provocan un encapsulamiento, cerrando su espacio, sin facilitar un vínculo con la comunidad y su historia social.

Desde sus historias, se reconoce que los Jardines son espacios contraculturales. Mejía (2013) afirma que estos proyectos moldean "una subjetividad rebelde, no sólo como contestación y resistencia, sino en la elaboración de propuestas alternativas para transformar sus entornos” (2013, p. 7). Estas prácticas son opuestas a una política tecnocrática que tiende a la homogeneización.

Estas experiencias que desarrollan los Jardines Comunitarios se presenta como una práctica contrahegemónica frente a lo que Fernandez (2001) señala como el "desfondamiento de la escuela"; es decir, la escuela se deslegitima y no tiene sentido en la formación de los niños y niñas, sino más bien tiende a normalizar y a burocratizar el sentido de lo pedagógico.

Por otra parte, se puede mencionar que los jardines comunitarios han resignificado el concepto de lo público. En su proyecto educativo existe un vínculo con la comunidad, "tema complejo en tiempos neoliberales, donde, así como se niega al sujeto, se niega la comunidad” (BUSTOS, 2012, p. 40). Sin duda, la acción que desarrollan en sus territorios es un aporte frente a comunidades fracturadas que han sido permeadas por el individualismo y por problemas sociales. Desde esta perspectiva, lo público implica reconstruir comunidad, donde ese espacio "no es solamente de todos, sino para todos y, por lo mismo, abierto siempre al otro en cuanto a otro. Es el espacio de la justicia” (CULLEN, 2008, p. 165).

Por último, se requiere también planificar una política para la primera infancia desde abajo hacia arriba. Desde esta perspectiva, la historia, las prácticas pedagógicas, un proyecto educativo centrado en la niñez y en su comunidad deberían incidir y ser escuchadas por los diseñadores de política educativa, en un escenario donde la educación de los niños y niñas tiende a la institucionalización. Además, frente a la rigidez impuesta por las prácticas escolarizadas en la primera infancia, los Jardines Comunitarios han avanzado hacia una educación humanista y emancipadora. Bajo esta mirada, no son sólo números en la política de cobertura, sino que han logrado responder a una pregunta fundamental en la educación: para qué educar y la respuesta a esa pregunta encuentra sentido en la comunidad. 


\section{Notas}

1. Principalmente Caritas y organismos internaciones como Unicef, Unesco y Programa Mundial de Alimentos (PMA).

2. Esta función antes la cumplía Junta Nacional de Jardines Infantiles, por lo que se queda sólo en su papel de proveedora de servicio.

3. Integra fue un espacio paliativo para el desempleo que se creó en Dictadura y contrataban a trabajadoras del Programa de Empleo Mínimo (PEM) y del Programa Ocupacional para Jefes de Hogar (desde 1983), sin mayor formación.

4. Según cifras del Ministerio de Educación, de 446 Jardines Infantiles JUNJI, fueron presentadas 255 solicitudes para reconocimiento oficial. Solo 93 Jardines Infantiles cuentan con reconocimiento oficial del Estado. En cambio, los Jardines Infantiles VTF no cuentan con las condiciones mínimas para iniciar la postulación a reconocimiento oficial del Estado en SECREDUC (MINEDUC, 2017).

5. Subsidio que se recibe por estudiante. Su propósito es la competencia entre las escuelas municipales y subvencionadas. En Chile, ha provocado una desigualdad.

6. Neoliberalismo se entiende como una organización particular de la relación entre la economía y sociedad en el marco capitalista. Esta relación implica una mercantilización de todas las esferas de la sociedad, lo que se traduce en una privatización de los servicios públicos, estado subsidiario y desregulación de la economía.

7. Este Programa Alternativo cuenta con cinco programas educativos: Jardín Familiar; Jardín Laboral; Jardín en Comunidades Indígenas; Programa de Mejoramiento de Atención a la Infancia (PMI); y los Centros Educativos Culturales de la Infancia (CECI).

8. Desde el año 1996, algunos Jardines Infantiles Comunitarios se han agrupado en la Red de Centros Comunitarios de Educación (Red CEC), en que participan 12 jardines infantiles de la Región Metropolitana. Además, existe un número indeterminado de centros educativos que se reconocen de la matriz comunitaria.

\section{Referencias}

ALBORNOZ, R. Los Centros de Desarrollo Infantil Comunitarios. Crecer juntos para la primera infancia. Encuentro regional de políticas integrales 2011. Unicef: Buenos Aires, 2012, p. 12-13.

ANCHETA, A.; LÁZARO, L. M. El derecho a la educación y atención de la primera infancia en américa latina. Revista Educación XXI, Madrid, v. 16, n. 1, p. 105-121, 2013.

ARAUJO, M. C.; LÓPEZ-BOO, F.; PUYANA, J. M. Panorama sobre los servicios de desarrollo infantil en América Latina y el Caribe. Washington: Banco Interamericano del Desarrollo, 2013.

BALL, S. J.; YOUDELL, D. La privatización encubierta de la educación pública. Londres: Instituto de Educación, Universidad de Londres, 2008.

BERLINSKI, S. Y; SCHADY, N. (eds.). Los primeros años: El bienestar infantil y el papel de las políticas públicas. Washington: Banco Interamericano de Desarrollo, 2015.

BUSTOS, L. Algunas claves para adentrarse en las avenidas de la gestión cultural y la animación sociocultural. In: Colectivo Paulo Freire. Prácticas, caminos y saberes para construir educación popular hoy. Santiago: Quimantu, 2012.

CABALUZ, . F. Pedagogías críticas latinoamericanas y filosofía de la liberación: Potencialidades de un diálogo teórico-político. Revista Educación y Educadores, v. 19, n. 1, p, 67-88, 2016. http://doi.org/10.5294/ edu.2016.19.1.4

CHILE. Ley n. 20.370. Establece la Ley General de Educación, de 17 de agosto de 2009. Disponible en: https:// www.leychile.cl/Navegar?idNorma=1006043. Acceso en: 16 ago. 2017. 
CULLEN, C. Perfiles ético-políticos de la educación. Buenos Aires: Paidos, 2004.

CULLEN, C. Critica a las razones de educar. Buenos Aires: Paidos, 2008.

CUSSIÁNOVICH, A.; FIGUEROA, E. Participación protagónica ¿Ideología o cambio de paradigma? In: LIEBEL, M.; MARTINEZ, M. (coords.). Infancia y derechos humanos. Hacia una ciudadanía participantes y protagónica. Lima: Instituto de Formación para Educadores de Jóvenes, Adolescentes y Niños Trabajadores de América Latina y el Caribe, 2009.

DIPRES [DIRECCION DE PRESUPUESTOS]. Informe final de evaluación programas gubernamentales (EPG). Programa educativo alternativo de atención del párvulo, 2017. Disponible en: http://www.dipres.gob. cl/597/articles-163120_informe_final.pdf. Acceso en: 9 jun. 2018.

FERNANDEZ, A. M. Instituciones estalladas. Buenos Aires: Eudeba, 2001.

FRASER, N. Iustitia Interrupta. Reflexiones críticas desde la posición “postsocialista”. Bogotá: Siglo del Hombre Editories/Universidad de los Andes, 1997.

FREIRE, P. Pedagogía de la esperanza: Un encuentro con pedagogía del oprimido. México: Siglo XXI, 1993.

FUJIMOTO-GÓMEZ, G. La educación no formal. Experiencias Latinoamericanas de atención a la infancia. La neoescolarización como alternativa. Congreso Mundial de Lecto Escritura, Valencia, 2000. Disponible en: http://www.waece.org/web_nuevo_concepto/textos/d141.pdf. Acceso en: 8 abr. 2018.

FULLAN, M.; HARGREAVES, A. La escuela que queremos. Los objetivos por los que vale la pena luchar. México: Amorrortu Editores, 2000.

GARCÉS, M. La revolución social en el Chile de Allende: Treinta años después La revolución de los pobladores, treinta años después. LASA, XXIV International Congress, Dallas, Texas, 27-29 mar. 2003.

GRUNNINGER, S. Las ONGs durante la Transición Chilena: Un análisis de su respuesta ideológica frente a su incorporación en políticas sociales de índole neoliberal. Revista Mad. Departamento de Antropología, Universidad de Chile, n. 9, sep. 2003.

KAMERMAN, S. B. A global history of early childhood education and care. EFA Global Monitoring Report 200. Strong foundations: Early childhood Care and Education. Unesco, 2006.

MEJÍA, M. R. La educación popular: Una construcción colectiva desde el sur y desde abajo. Seminario Internacional de Educación Popular, CINEP/Alcaldía Mayor de Bogotá, Bogotá, 13-15 feb. 2013.

MINEDUC [MINISTERIO DE EDUCACIÓN]. Hoja de Ruta. Definiciones de política para una educación parvularia de calidad. Mineduc: Santiago, 2016

MINEDUC [MINISTERIO DE EDUCACIÓN]. Balance de gestión integral, 2017. Junta Nacional de Jardines Infantiles. Cuenta Pública 2014-2018. Disponible en: http://www.dipres.gob.cl/597/articles-172574_doc_pdf. pdf. Acceso en: 115 ene. 2019.

MUÑOZ, G. Formación de capital social en dos asentamientos urbanos de la comuna de Cerrillos, Parcela 21 y Villa Los Presidentes (Región Metropolitana 1960-1998). Investigación y Crítica, v. 1, n. 1, p. 229-286, 1999.

PERALTA, V. Programas no formales en la educación parvularia (aportes y Proyecciones). Chile: JUNJI, 2018. 
PINTO, R. El currículum crítico. Una pedagogía transformativa para la educación latinoamericana. Santiago: Ediciones Universidad Católica de Chile, 2007.

RAMÍREZ OTÁlVARO, O. P. (eds.). El derecho a la educación en la primera infancia. OMEP Región América Latina. Medellín, 2015.

REDONDO, P. Políticas en debate: La atención educativa de la primera infancia en la Argentina. Propuesta Educativa, año 21, n. 37, p. 6-16, 2012.

REIS MONTEIRO, A. La revolución de los derechos del niño, Madrid: Editorial Popular, 2008.

SALAZAR, G. 17 años de lucha. Movimientos sociales bajo la dictadura militar, 2013. Disponible en: https:// www.youtube.com/watch?v=LOFJ56cihBA. Acceso en: 6 ene. 2016.

SOLIMANI, A. Capitalismo a la chilena y la prosperidad de las elites. Santiago: Catelonia, 2012.

STAKE, R. Investigación con estudio de casos. Madrid: Ediciones Morata, 1998.

TOKMAN, A. Radiografía de la educación parvularia chilena: Desafíos y propuestas. Santiago: Serie de Políticas Públicas UDP, 2010.

VALDÉS, T.; WEINSTEIN, M. Organizaciones pobladoras y construcción democrática en Chile. Chile, Programa FLACSO, n. 434, oct. 1989.

YOUNG, M. E.; FUJIMOTO-GÓMEZ, G. Desarrollo Infantil Temprano: lecciones de los programas no formales. Acción Pedagógica, v. 13, n. 2, p. 186-198, 2004,

\section{Sobre la Autora}

Graciela Muñoz Zamora es educadora de Párvulos de la Universidad Metropolitana de Ciencias de la Educación. Doctora en Educación de la Universidad de Playa Ancha de Ciencias de la Educación. Miembro del Foro por el Derecho de Educación - Chile y CEDI. Líneas de investigación: Imaginarios de la Infancia; Políticas de La Primera infancia; e Identidad Docente.

Recibido: 24 jul. 2018 Aceptado: 20 jun. 2020 